\title{
Some properties of absolutely continuous invariant measures on an interval
}

\author{
FRANÇOIS LEDRAPPIER† \\ Laboratoire de calcul des probabilités, Université Paris, France
}

(Received 20 August 1980 and revised 7 October 1980)

\begin{abstract}
$A$ bstract. We are interested in ergodic properties of absolutely continuous invariant measures of positive entropy for a map of an interval. We prove a Bernoulli property and a characterization by some variational principle.
\end{abstract}

We consider (piecewise) $C^{2}$ maps from an interval into itself. Sufficient conditions have been given for the existence of an invariant measure which is absolutely continuous with respect to the Lebesgue measure: we shall write this a.c.i.m. (See Bowen [4] for discussion and references; Misiurewicz [15], Jakobson [10] and Szlenk [33] for recent results.) We want to study the ergodic properties of such an a.c.i.m. in the case when the expansivity is not uniform, but only asymptotic: We consider only maps which are not flat at their critical points.

We show that for an a.c.i.m. weak mixing and positive entropy imply a Bernoulli property: each finite partition in intervals is weak Bernoulli. This result was known in the uniformly expansive case (Bowen [3]). We also show for a $C^{2}$ map $f$ that a.c.i.m. of positive entropy are characterized by a variational principle: an ergodic measure $\mu$ of positive entropy $h$ is an a.c.i.m. if and only if it satisfies Rohlin's formula: $h=\int \log \left|f^{\prime}\right| d \mu$. This result is also true in the piecewise $C^{2}$ case if the measure is non-degenerate (see definition 1.3 below). It was known only in some particular cases, like $\beta$-transformations (Walters [34], Hofbauer [8]). This characterization leads to criteria for the existence of an a.c.i.m. We give an example: if there is a set of positive measure of regular points with positive characteristic exponent, then there exists an a.c.i.m.

In order to prove these results, we construct the local unstable manifold in some natural extension of the system. Much more general results about local unstable manifolds for a non-invertible map are announced by Ruelle and Shub [29], and I am very grateful to $D$. Ruelle ior having acquainted me with this idea.

Then we prove the absolute continuity of the unstable foliation, from which ergodic properties follow. Absolute continuity of the unstable foliation is the key property of Sinaī's measure in several examples (Anosov systems [31] and flows

† Address for correspondence: Dr F. Ledrappier, Laboratoire de calcul des probabilités, Université Paris VI Tour 56, 4 Place Jussieu, 75230 Paris cedex 05, France. 
[32], Axiom A attractors for diffeomorphisms [24] and flows [5], Lorenz' attractor [6]) and also of invariant smooth measure for diffeomorphisms (Pesin [18]). Ruelle [26] proposed that measures describing turbulence are measures with that property. This paper shows that in dimension one, even in the non-uniform case, this proposition is consistent with other natural notions such as being an a.c.i.m. of positive entropy or satisfying a variational principle.

Part of this work was done while visiting the University of Rome, with the support, hospitality and interest from the statistical mechanics Roman team.

\section{Notations and results}

We shall say that a real function $f$ on an interval $[a, b]$ satisfies condition $\mathrm{C}$, or is a C-map, if:

Condition $C$. The map $f$ is continuously differentiable and its derivative $f^{\prime}$ has the following properties:

$\left(\mathrm{C}_{1}\right) f^{\prime}$ satisfies a Hölder condition of order $\varepsilon, \varepsilon>0$.

$\left(\mathrm{C}_{2}\right)$ There are only a finite number of critical points. We denote them by $a \leq a_{1}<a_{2} \cdots<a_{n} \leq b$ with $f^{\prime}\left(a_{i}\right)=0$ for $0<i \leq n$.

$\left(\mathrm{C}_{3}\right)$ There exist positive numbers $k_{i}^{-}\left(k_{i}^{+}\right)$such that

$$
\left|\log \frac{\left|f^{\prime}(x)\right|}{\left|x-a_{i}\right|^{k_{i}^{-(+)}}}\right|
$$

is bounded in a left (right) neighbourhood of $a_{i}$.

Let $I$ be the unit interval. A map $f$ from $I$ into itself will be called piecewise condition C or a PC-map if there exists a finite partition $0<b_{1}<b_{2} \cdots<b_{m}<1$ such that $f$ is a C-map from $\left[b_{j}, b_{j+1}\right]$ into $I$, for any $j$.

A probability measure $\mu$ on $I$ is said to be invariant if $\mu\left(f^{-1} A\right)=\mu(A)$ for every Borel set $A$, and ergodic if it is invariant and every invariant set has measure 0 or 1. The entropy $h(\mu)$ of an invariant measure is a positive number (cf. [23]); the entropy is 0 if and only if the map $f$ is $\mu$-a.e. invertible. Conversely, a finite partition $P$ in a system $(X, f, \mu)$ defines a weak Bernoulli process [16] if, for any $\varepsilon>0$, there exists an $n$ such that for any $m, p$

$$
\sum_{a, b}|\mu(a \cap b)-\mu(a) \mu(b)|<\varepsilon,
$$

where the sum extends over all atoms of the partitions

respectively.

$$
\bigvee_{i=0}^{m-1} f^{-i} P \text { and } \bigvee_{i=m+n}^{m+n+p} f^{-i} P
$$

The main results of this paper are the following.

THEOREM 1. Let $f$ be a PC-map, $Q$ the partition of I defined by the critical points and the points of discontinuity, $\mu$ an ergodic a.c.i.m. of positive entropy. If $\mu$ is ergodic for $f^{k}$, for all $k>0$, then $Q$ defines a weak Bernoulli process. In any case, there exists a $k_{0} \geq 1$ such that the partition $\bigvee_{i=0}^{k_{0}-1} f^{-i} Q$ defines a weak Bernoulli process with $f^{k_{0}}$ on any ergodic component. 
We remark that, by introducing new discontinuities, any finite partition in intervals can play the role of $Q$ in theorem 1 .

Corollary 2. Let $f$ be a PC-map, $\mu$ an ergodic a.c.i.m., A the $\sigma$-field of Borel sets on I. The tail $\sigma$-field $\bigwedge_{n} f^{-n} \mathscr{A}$ is either finite or equal to $\mathscr{A}$, according to whether $\int \log \left|f^{\prime}\right| d \mu$ is strictly positive or zero.

THEOREM 3. Let f be a C-map. An ergodic invariant measure $\mu$ with positive entropy is an a.c.i.m. if and only if Rohlin's formula (1.1) is true:

(1.1) $h(\mu)=\int \log \left|f^{\prime}\right| d \mu$.

Theorem 3 is also valid for PC-maps if one considers only measures which are non-degenerate (definition 1.3 below).

Corollary 4. Let $f$ be a PC-map, $\mu$ an a.c.i.m. Almost all ergodic components of $\mu$ with positive entropy are still absolutely continuous. Therefore there can be essentially only a countable number of different ergodic components with positive entropy.

A counterexample to theorem 3 for a $C^{1}$ map can be obtained from a construction of Bowen's [2] (this remark is due to L. S. Young). As an example of an application of theorem 3 , let us define a point $x$ in $I$ to be a regular point if the following properties hold:

$\left(\mathrm{R}_{1}\right)$ The sequence of measures $(1 / n) \sum_{i=0}^{n-1} \delta_{f^{i} x}$ converges vaguely towards an ergodic measure $\mu_{x}\left(\delta_{y}\right.$ denotes the Dirac measure at $\left.y\right)$.

$\left(\mathrm{R}_{2}\right)$ The sequence of numbers $(1 / n) \log \left|f^{n^{\prime}}(x)\right|$ converges towards $\lambda_{x}$.

$\left(\mathbf{R}_{3}\right)$ We have $\lambda_{x}=\int \log \left|f^{\prime}\right| d \mu_{x}$.

A regular point is called positive regular if $\lambda_{x}>0$. The set of (positive) regular points is measurable ([9], p. 363).

In some sense, orbits of regular points are the very orbits an experimentalist wants to observe. One may argue that he can do so only if regular points have a positive Lebesgue measure. Our criterion deals with that condition and was suggested by the numerical evidence of [1].

THEOREM 5. Let $f$ be a C-map from I into itself. The set of positive regular points has a positive Lebesgue measure if and only if there exists an a.c.i.m. of positive entropy.

Theorem 5 follows from theorem 3 and proposition 6 .

Proposition 6. Let f be a C-map. For Lebesgue almost every regular point, we have $h\left(\mu_{x}\right)=\max \left(0, \lambda_{x}\right)$.

The proofs of the main theorems use the construction of an invertible extension of $f$ as follows: let $f$ be a PC-map, $0=a_{0}<a_{1}<\cdots<a_{n+1}=1$ be the sequence of critical points and points of discontinuity. Let $Z$ be the set of sequences of $\{0,1, \ldots, n, n+1\}, z=\left\{z_{1}, z_{2}, \ldots, z_{n}, \ldots\right\}$. 
(1.2) We call the system $(Y, f)$ the extension of $(I, f)$, where $Y$ is the subset of $I \times Z$ composed of all the pairs $(x, z)$ such that, for any $m$, there exists $x_{m}$ in $I$ satisfying

$$
f\left(x_{1}\right)=x, \quad f\left(x_{m}\right)=x_{m-1}, \quad a_{z_{m}} \leq x_{m}<a_{z_{m}+1},
$$

and $f$ is defined by

$$
f(x, z)=\left(x^{\prime}, z^{\prime}\right), \quad \text { with } x^{\prime}=f(x), z_{n}^{\prime}=z_{n-1}
$$

for $n>1$ and $z_{1}^{\prime}$ is the only index for which $a_{z i} \leq x<a_{z_{1}^{\prime}+1}$.

It is easy to check that the map $f$ on $Y$ is invertible, its inverse $f^{-1}$ being given by $f^{-1}(x, z)=\left(x^{\prime \prime}, z^{\prime \prime}\right)$, where $x^{\prime \prime}$ is the first element $x_{1}$ (see above) in the definition of $(x, z)$ belonging to $Y$, and $z_{n}^{\prime \prime}=z_{n+1}$. It is also clear that the projection $\Pi$ on $I$ commutes with the maps $f$ and that, for any invariant measure $\mu$ on $I$, there exists a unique invariant measure $\bar{\mu}$ on $Y$ whose image by $\Pi$ is $\mu$. If the measure $\mu$ is ergodic, so is $\bar{\mu}$. Put $\delta(y)=\inf _{i}\left|\Pi(y)-a_{i}\right|$.

Definition 1.3. We say that an invariant measure $\mu$ is non-degenerate if we have

$$
\liminf _{n}(1 / n) \log \delta\left(f^{-n} y\right)=0 \quad \bar{\mu} \text {-a.e. }
$$

Proposition 7. Let $f$ be $a \mathrm{C}$-map and $\mu$ an ergodic non-atomic invariant measure with $\int \log \left|f^{\prime}\right| d \mu>-\infty$, or let $f$ be a PC-map and $\mu$ an a.c.i.m. In both cases, the measure $\mu$ is non-degenerate.

For a non-degenerate measure we can construct the local unstable manifold.

THEOREM 8. Let $f$ be a PC-map and $\mu$ a non-degenerate invariant ergodic measure. Let $0<\chi<\int \log \left|f^{\prime}\right| d \mu$. Then there exist on $Y$ four measurable functions $\alpha, \beta, \gamma, \bar{\gamma}$ and a constant $D$ such that:

(i) $\alpha>0,1<\beta<\infty, 0<\gamma<\infty \quad \bar{\mu}$-a.e.;

(ii) let $y=(x, z)$ and $|t|<\alpha(y)$; the point $y_{t}=(x+t, z)$ lies in $Y$;

(iii) $\left|\Pi\left(f^{-n} y\right)-\Pi\left(f^{-n} y_{t}\right)\right| \leq \beta(y) \exp \left(-\chi^{n}\right)|t|$;

(iv) for any $n$,

$$
\begin{gathered}
\frac{1}{D} \leq \frac{f^{\prime}\left(\Pi\left(f^{-n} y\right)\right)}{f^{\prime}\left(\Pi\left(f^{-n} y_{t}\right)\right)} \leq D ; \\
\gamma(y) \leq \prod_{n=1}^{\infty} \frac{f^{\prime}\left(\Pi\left(f^{-n} y\right)\right)}{f^{\prime}\left(\Pi\left(f^{-n} y_{t}\right)\right)}=\Delta\left(y, y_{t}\right) \leq \bar{\gamma}(y) .
\end{gathered}
$$

Let us consider the equivalence relation on $Y$ defined by the projection on $Z$. Theorem 8 says that classes are made of open subsets of the interval, so that this equivalence relation defines a 'measurable foliation' of dimension one. The classes of that equivalence relation also define a measurable partition $\xi$. Absolute continuity of the foliation (Sināi [30]) is a property of conditional measures, or Rohlin decomposition, with respect to $\xi$. 
THEOREM 9. Let $f$ be a PC-map and $\mu$ a non-degenerate invariant ergodic measure such that $h(\mu)=\int \log \left|f^{\prime}\right| d \mu>0$. Let $q(y, \cdot)$ be a Rohlin decomposition of $\bar{\mu}$ for the partition $\xi$. Then for $\bar{\mu}$ almost all $y$, the measure $q\left(y, \Pi^{-1}, \cdot\right)$ on $I$ is absolutely continuous with respect to the Lebesgue measure. We even know the densities of the conditional measures (proposition 3.6).

We first prove theorem 8. Local unstable manifolds in the non-uniform case were first constructed by Pesin [18]. Further progress has been made by Ruelle [27], Fathi, Herman \& Yoccoz [7], Katok \& Strelcyn [11], Ruelle [28], Mañé, Ruelle \& Shub [29]. Our result is contained in the union of all those results and it is much simpler. We give a proof of it for the sake of completeness and notations, and in order to show the role of the different conditions. We then prove theorem 9. The proof uses mainly the construction of a measurable increasing partition, as Pesin did in [19], and the concavity of $\log$, as in [12]. We then make some remarks which lead to propositions 6 and 7 and, finally, combine our proofs to obtain the other announced results. We shall use basic facts from Rohlin's papers [21] and [23] without any other particular reference.

\section{Local unstable manifold theorem}

We consider a PC-map and we keep the notations of $\S 1$. We put $F(y)=\left|f^{\prime}\left(\Pi\left(f^{-1} y\right)\right)\right|$. We first prove some lemmas.

LEMMA 2.1. Let $f$ be a PC-map, $y$ a point in $Y, y=(x, z)$ and $F(y) \neq 0$. There exists a constant $D$ such that, if $|t|<(1 / 2 D) \delta\left(f^{-1} y\right) F(y)$, then there exists a point $x_{\imath}^{\prime}$ in $I$ such that

$$
f\left(x_{t}^{\prime}\right)=x+t, \quad a_{z_{1}}<x_{t}^{\prime}<a_{z_{1}+1}, \quad 1 / D \leq\left|f^{\prime}\left(x_{t}^{\prime}\right)\right| / F(y) \leq D .
$$

Proof. Let us consider a point $x$ in $I$ and $i$ the index such that $\left|x-a_{i}\right|=\delta(x)$. If $\left|x-x^{\prime}\right| \leq \frac{1}{2} \delta(x)$, then $\frac{1}{2} \leq\left(x-a_{i}\right) /\left(x^{\prime}-a_{i}\right) \leq \frac{3}{2}$ and, by $C_{3}$ if $a_{i}$ is a critical point, by $C_{1}$ otherwise, we can find a uniform $D$ such that $(1 / D) \leq f^{\prime}\left(x^{\prime}\right) / f^{\prime}(x) \leq D$. Therefore for $y$ in $Y$ and $t$ real, the conclusions of lemma 2.1 are true as soon as $y+t$ lies in the image by $f$ of the interval $\left[\Pi\left(f^{-1} y\right)-\frac{1}{2} \delta\left(f^{-1} y\right), \Pi\left(f^{-1} y\right)+\frac{1}{2} \delta\left(f^{-1} y\right)\right]$. The lemma follows because $\left|f^{\prime}\right|$ is bounded from below by $F(y) / D$ on this interval.

Let us now define $\lambda(y)=(1 / 2 D) \delta\left(f^{-1} y\right) F(y)$ and let us choose a real $C^{3}$ function $\theta$ on the real line such that $\theta(t)=1$ if $|t| \leq \frac{1}{2}, 0$ if $|t| \geq 1$. For any $y$ in $Y$ with $F(y) \neq 0$ define the real function $G_{y}$ by

$$
G_{y}(t)=t / F(y)+\theta(t / \lambda(y)) \cdot\left(x_{t}^{\prime}-\Pi\left(f^{-1} y\right)-t / F(y)\right),
$$

where $x_{t}^{\prime}$ is given by lemma 2.1 .

LEMMA 2.2. (i) $G_{y}(t)=x_{t}^{\prime}-\Pi\left(f^{-1} y\right)$ for $|t|<\frac{1}{2} \lambda(y) ;\left|G_{y}^{\prime}(0)\right|=1 / F(y)$.

(ii) There exists $K>1$ such that, for any $s, t$ reals, we have

$$
\left|G_{y}^{\prime}(s)-G_{y}^{\prime}(t)\right| \leq \frac{K}{(F(y))^{2}}|s-t|^{\varepsilon} .
$$


Proof. Property (i) is immediate. Let us choose $M$ such that $\left|f^{\prime}(t)-f^{\prime}(s)\right| \leq M|t-s|^{\varepsilon}$ if $s, t$ belong to the same interval. To prove (ii), we compute $\left|G_{y}^{\prime}(t)-G_{y}^{\prime}(s)\right|$.

In the case when $|t|<\lambda(y)$ and $|s|>\lambda(y)$, we have

$$
\begin{aligned}
\mid G_{y}^{\prime}(s) & -G_{y}^{\prime}(t) \mid \\
& \leq \theta(t / \lambda(y))\left|\frac{1}{f^{\prime}\left(x_{t}^{\prime}\right)}-\frac{1}{F(y)}\right|+(1 / \lambda(y))\left|\theta^{\prime}(t / \lambda(y))\right|\left|x_{t}^{\prime}-\Pi\left(f^{-1} y\right)-t / F(y)\right|, \\
& \leq \frac{D M}{(F(y))^{2}} \lambda(y)^{\varepsilon} \theta(t / \lambda(y))+\frac{D M}{(F(y))^{2}} \frac{\lambda(y)^{\varepsilon}}{1+\varepsilon}\left|\theta^{\prime}(t / \lambda(y))\right| \quad \text { by } \mathrm{C}_{1} \text { and lemma 2.1, } \\
& <\frac{C}{(F(y))^{2}}|t-s|^{\varepsilon} \quad \text { for some constant } C .
\end{aligned}
$$

The estimation is quite similar if both $s$ and $t$ are smaller than $\lambda(y)$.

LEMMA 2.3. Let $(X, f, \mu)$ be a dynamical system, $g$ in $L^{1}(X, \mu)$. Then

$$
\lim (1 / n) g \circ f^{n}=0 \mu \text {-a.e. }
$$

Proof. This follows immediately from the pointwise ergodic theorem.

LEMMA 2.4. Let $\mu$ be a non-degenerate invariant measure such that $\int \log \left|f^{\prime}\right| d \mu$ is finite. Then $\lim _{n} \inf (1 / n) \log \lambda\left(f^{-n} y\right)=0 \bar{\mu}$-a.e.

Proof. This follows clearly from the non-degeneracy of the measure $\mu$ and lemma 2.3 applied to the integrable function $\log F$.

Let us now state the non-linear ergodic theorem ([27] theorem 5.1) for a family of one-dimensional maps.

THEOREM 2.5 (Non-linear ergodic theorem). Let $(X, \Sigma, \rho ; f)$ be a dynamical system and, for any $x$ in $X, G_{x}$ be a $C^{1+\theta}$ real function, $\chi<0$. We write $G_{x}^{n}=$ $G_{f^{n-1} x} \circ \cdots \circ G_{f x} \circ G_{x}$ and we suppose that $\log ^{+}\left\|G_{x}\right\|_{1, \theta}$ is integrable and that $\limsup _{n}(1 / n) \log \left|G_{x}^{n^{\prime}}(0)\right|<\chi \rho$-a.e. Then there exist measurable functions $\alpha(x), \beta(x)$ such that, if $|t|<\alpha(x),\left|G_{x}^{n}(t)\right| \leq \beta(x) \exp (n \chi)$ for all $n \geq 0$.

Proof of theorem 8. Let $f$ be a PC-map and $\mu$ a non-degenerate ergodic measure. Let us suppose that $\int \log \left|f^{\prime}\right| d \mu>0$ and let us choose $\chi, 0<\chi<\int \log \left|f^{\prime}\right| d \mu$. By (2.5) applied to $\left(Y, f^{-1}\right)$ and lemma 2.2 , there exist measurable functions on $Y$ $0<\alpha^{\prime}<\beta<+\infty \bar{\mu}$-a.e., such that, if $|t|<\alpha^{\prime}(y)$, then $\left|G_{y}^{n}(t)\right| \leq \beta(y) \exp (-n \chi)|t|$.

We now put

$$
\alpha(y)=\inf \left(\alpha^{\prime}(y), \lambda\left(f^{-n} y\right) \exp (n \chi) / 2 \beta(y), n \geq 0\right) .
$$

By lemma 2.4, $\alpha$ is positive almost everywhere. We have now for $|t|<\alpha(y)$, and any $n \geq 0$,

$$
\left|G_{y}^{n}(t)\right| \leq \beta(y) \exp (-n \chi)|t| \quad \text { and } \quad\left|G_{y}^{n}(t)\right|<\frac{1}{2} \lambda\left(f^{-n} y\right) .
$$

The sequence $\left\{x_{t}^{n+1}\right\}$ which we obtain by applying lemma 2.1 to $\left(f^{-n} y, G_{y}^{n}(t)\right)$ successively satisfies, by lemma 2.2 (i), $f\left(x_{t}^{n}\right)=x_{t}^{n-1}$ and therefore defines a unique 
point $y_{t}$ in $Y$; it clearly has the properties (ii), (iii), (iv) of theorem 8. Property (v) follows from (iii), (iv) and the Hölder condition on $f^{\prime}$.

3. Absolute continuity of the unstable foliation

We first show a preliminary lemma.

LeMMA 3.1. Let $m$ be a finite measure on $I$; for Lebesgue almost all $x$ in $I$, for all $\alpha<1, \lim \sup (1 / n) \log m\left[x-\alpha^{n}, x+\alpha^{n}\right]<0$.

Proof. We have only to prove the formula for rational $\alpha<1$. Let us call $\boldsymbol{A}_{k}$ the set of $x$ such that $m\left[x-\alpha^{k}, x+\alpha^{k}\right] \leq 2 k^{2} \alpha^{k}$, and let us cover $I \backslash A_{k}$ by intervals $C_{i}=$ $\left[x_{i}-\alpha^{k}, x_{i}+\alpha^{k}\right]$, in such a way that $x_{i} \notin A_{k}$ and any point of $I$ is at most in two different intervals $C_{i}$. If $\lambda$ denotes the Lebesgue measure, we have

$$
\lambda\left(I \backslash A_{k}\right) \leq \sum_{i} \lambda\left(C_{i}\right) \leq\left(1 / k^{2}\right) \sum_{i} m\left(C_{i}\right) \leq 2 / k^{2} .
$$

By the Borel-Cantelli lemma, $\lambda$-almost every point lies in $\lim _{k} \inf A_{k}$, and therefore satisfies

$$
\limsup _{n}(1 / n) \log m\left[x-\alpha^{n}, x+\alpha^{n}\right] \leq \lim \sup _{n}(1 / n) \log 2 n^{2} \alpha^{n}<0 .
$$

Lemma 3.1 allows us to construct Pesin's partition in the general case.

Proposition 3.2. Let $f$ be $a$ PC-map and $\mu$ a non-degenerate invariant ergodic measure, $\int \log \left|f^{\prime}\right| d \mu>0$. There exists a measurable partition $\eta$ of $Y$ with the following properties:

(i) the partition $\eta$ is increasing by $f, f^{-1} \eta>\eta$, and generates;

(ii) the entropy of $\mu$ is given by $h(\mu)=H\left(f^{-1} \eta / \eta\right)$;

(iii) the elements of $\eta$ are subsets of the elements of the partition $\xi$;

(iv) we have $\bar{\mu}$-a.e. $0<\int_{\eta(y)} \Delta\left(y, y^{\prime}\right) d y^{\prime}<\infty$, where $\eta(y)$ denotes the element of $\eta$ containing $y$ and denotes the natural Lebesgue measure on each element of $\eta$.

Proof. We shall first construct the partition and then check properties (i) to (iv). We consider the functions $\alpha, \beta$ and $\gamma$ given by theorem 8 .

We call $\Pi^{\prime}$ the projection from $Y$ into $Z$. Let us consider $\alpha_{0}, \beta_{0}$ such that $\bar{\mu}\left(\left\{\alpha>\alpha_{0}, \beta<\beta_{0}\right\}\right)>0$ and a measurable map $\rho$ from $B=\Pi^{\prime}\left(\left\{\alpha>\alpha_{0}, \beta<\beta_{0}\right\}\right)$ into $\left\{\alpha>\alpha_{0}, \beta<\beta_{0}\right\}$ which is a section of $\Pi^{\prime}$. We consider $A_{s}$, the set of $y$ such that $\Pi^{\prime}(y)$ lies in $B$ and $\left|\Pi(y)-\Pi \rho\left(\Pi^{\prime}(y)\right)\right|<s$. By modifying $\rho$ if necessary, we may suppose that $\bar{\mu}\left(A_{\alpha_{0} / 2}\right)>0$.

For any $0<\tau<1$, any integer $n$, consider $A_{s}^{\tau, n}$, the set of $y$ such that $\Pi^{\prime}(y)$ lies in $B$ and ||$\Pi(y)-\Pi \rho\left(\Pi^{\prime}(y)\right)|-s|<\tau^{n}$. By lemma 3.1 we can choose $s<\alpha_{0}$ such that

$$
\bar{\mu}\left(A_{s}\right)>0 \text { and } \sum_{n} \bar{\mu}\left(A_{s}^{\tau, n}\right) \text { is finite for all } \tau<1 .
$$


For such an $s$ define $\eta$ as the partition generated by the images of $A_{s}$ and of the partition $Q$ determined by the $a_{i}$ :

$$
\eta=\bigvee_{i=0}^{\infty} f^{i}\left(\left\{A_{s}, Y \backslash A_{s}\right\} \vee Q\right) .
$$

From the definition it is immediate that $\eta$ is a measurable partition, increasing by $f$, and that its elements are contained in the elements of $\xi$, because $\xi=\bigvee_{i=1}^{\infty} f^{i} Q$.

Furthermore, we have a generating partition because, if two points $y_{1}$ and $y_{2}$ are such that all their images $f^{i} y_{1}$ and $f^{i} y_{2}$ lie in the same element of $\left\{\boldsymbol{A}_{s}, Y \backslash A_{s}\right\} \vee \xi$, we have almost everywhere for an infinite number of $n_{i}, f^{n_{i}} y_{1}$ and $f^{n_{i}} y_{2}$ in the same local unstable manifold with $\alpha>\alpha_{0}, \beta<\beta_{0}$; therefore $\left|\Pi\left(y_{1}\right)-\Pi\left(y_{2}\right)\right|<\beta_{0} \exp \left(-\chi n_{i}\right) \alpha_{0}$, and $y_{1}$ and $y_{2}$ must coincide. This means that almost any two points are separated by $\vee f^{n} \eta$. The partition $\eta$ is therefore the future of a finite generating partition $P$, and the entropy is given by

$$
h(\mu)=H\left(P / \bigvee_{i=1}^{\infty} f^{i} P\right)=H\left(f^{-1} \eta / \eta\right) .
$$

We have now only to verify property (iv), which divides into two properties. The strict positivity of the integral follows from:

LEMMA 3.4. For $\bar{\mu}$-almost any $y$ in $Y, \eta(y)$ contains an open neighbourhood of $y$ in $\xi(y)$.

Proof of lemma 3.4. Let us call $E$ the intersection of $A_{s}^{\tau, n}, n>1 ; E$ is the 'boundary' of $A_{s}$. Let us denote the distance from $y$ to $E$ in $\xi(y)$ by $\omega(y)$. By (3.3) for any $\tau<1$, we have that $\sum_{n} \bar{\mu}\left(\left\{\omega\left(f^{-n} y\right)<\tau^{n}\right\}\right)$ is finite. By the Borel-Cantelli lemma, it follows that, for any $\chi>0$, the function $\zeta(y)=\inf \left(\omega\left(f^{-n} y\right) \exp (n \chi), n \geq 0\right)$ is strictly positive $\bar{\mu}$-a.e. Therefore, let us consider $y$ in $Y$ and a real $t,|t|$ smaller than $\alpha(y), \delta(y)$, and $\zeta(y) / 2 \beta(y)$. By theorem 8 , there exists a point $y_{t}^{\prime}$ in $Y$, in the same element of $\xi=\bigvee_{i=1}^{\infty} f^{-i} Q$, and such that

$$
\left|\Pi\left(f^{-n} y\right)-\Pi\left(f^{-n} y_{t}^{\prime}\right)\right| \leq \beta(y) \exp (-n \chi)|t|<\frac{1}{2} \zeta(y) \exp (-n \chi)<\omega\left(f^{-n} y\right) .
$$

Therefore $f^{-n} y$ and $f^{-n} y_{t}^{\prime}$ are both in $A_{s}$ or both in $Y \backslash A_{s}$ for all $n \geq 0$. Since $|t|<\delta(y)$, $y$ and $y_{t}^{\prime}$ are also both in the same element of $Q$. Finally, for almost all $y$, we have found an open set of reals $t$ such that $y_{t}^{\prime}$ belongs to $\eta(y)$, and that proves lemma 3.4 .

The finiteness of the integral in property (iv) follows from theorem 8 (v) if $y$ is in $A_{s}$, because $\Delta$ is bounded on the local unstable manifold, which contains $\eta(y)$. If $y$ is not in $\boldsymbol{A}_{s}$, by ergodicity, there exists a positive $k$ such that $f^{-k} y$ is in $A_{s}$ and then $\eta(y)=f^{k}\left(\left[f^{-k} \eta\right]\left(f^{-k} y\right)\right)$. We have therefore

$$
\begin{aligned}
\int_{\eta(y)} \Delta\left(y, y^{\prime}\right) d y^{\prime} & =\int_{f^{k}\left(\left[f^{-k} \eta\right]\left(f^{-k} y\right)\right)} \Delta\left(f^{-k} y, f^{-k} y^{\prime}\right) \prod_{i=1}^{k} \frac{f_{\Pi}^{\prime}\left(f^{-i} y\right)}{f_{\Pi}^{\prime}\left(f^{-i} y^{\prime}\right)} d y^{\prime} \\
& =\prod_{i=1}^{k}\left|f^{\prime}\left(f^{-i} y\right)\right| \int_{\left[f^{-k} \eta\right]\left(f^{-k} y\right)} \Delta\left(f^{-k} y, y^{\prime}\right) d y^{\prime}
\end{aligned}
$$


and this integral is finite because $\left[f^{-k} \eta\right]\left(f^{-k} y\right)$ is contained in the local unstable manifold of the point $f^{-k} y$; this achieves the proof of proposition 3.2.

We recall also the following classical lemma.

LEMMA 3.5. Let $(X, \mu, f)$ be a dynamical system and $g_{i}, i=1,2,3$, be functions related by $g_{1}=g_{2}+g_{3} \circ f-g_{3}$, with $g_{2}$ and $g_{1}^{+}$integrable. Then we have

$$
\lim (1 / n) g_{3} \circ f^{n}=\hat{g}_{1}-\hat{g}_{2}=0 \mu \text {-a.e., }
$$

where $\hat{\mathrm{g}}$ denotes the pointwise limit of ergodic averages of $\mathrm{g}$.

Proof. The first equality comes by applying $f^{i}$ to the given relation and averaging. On the other hand, the random variables $(1 / n) g_{3} \circ f^{n}$ converge in measure towards 0 . Therefore, the almost everywhere limit has to be 0 .

The next proposition expresses the absolute continuity of the unstable foliation.

Proposition 3.6. Let $f$ be a PC-map and $\mu$ a non-degenerate invariant ergodic measure such that $\int \log \left|f^{\prime}\right| d \mu=h(\mu)>0$. The Rohlin decomposition for the measure $\bar{\mu}$ with respect to the partition $\eta$ of proposition 3.2 is given by $q(y, B)$, for $y$ in $Y$ and $B$ a measurable subset,

$$
q(y, B)=\frac{\int_{B \cap \eta(y)} \Delta\left(y, y^{\prime}\right) d y^{\prime}}{\int_{\eta(y)} \Delta\left(y, y^{\prime}\right) d y^{\prime}} .
$$

Proof. Let us remark first that $q(y, \cdot)$ in (3.6) is a probability measure such that $q(y, \eta(y))=1$ and that, if $\eta\left(y_{1}\right)=\eta\left(y_{2}\right), q\left(y_{1}, \cdot\right)$ is equal to $q\left(y_{2}, \cdot\right)$ by multiplying both factors by $\Delta\left(y_{1}, y_{2}\right)$.

Let us consider the Rohlin decomposition for the measure $\mu$ with respect to the partition $\eta, p(y, \cdot)$, and we take an integer $n$. We have, by proposition 3.2 (ii),

$$
n h(\mu)=H\left(f^{-n} \eta / \eta\right)=-\int \log p\left(y,\left[f^{-n} \eta\right](y)\right) \bar{\mu}(d y) .
$$

In addition, the following relation is true:

$$
-\int \log q\left(y,\left[f^{-n} \eta\right](y)\right) \bar{\mu}(d y)=\int \log \left|f^{n^{\prime}}\right| d \bar{\mu}=n \int \log \left|f^{\prime}\right| d \mu .
$$

(3.8) follows from lemma 3.5 and the following relation:

$$
q\left(y,\left[f^{-n} \eta\right](y)\right)=\frac{\int_{\left[f^{-n} \eta\right](y)} \Delta\left(y, y^{\prime}\right) d y^{\prime}}{\int_{\eta(y)} \Delta\left(y, y^{\prime}\right) d y^{\prime}},
$$


which becomes by letting $k(y)=\int_{\eta(y)} \Delta\left(y, y^{\prime}\right) d y^{\prime}$,

$$
\begin{aligned}
q\left(y,\left[f^{-n} \eta\right](y)\right) & =\frac{1}{k(y)} \int_{f^{-n}\left(\eta\left(f^{n} y\right)\right)} \Delta\left(f^{n} y, f^{n} y^{\prime}\right) \frac{f^{n^{\prime}}\left(y^{\prime}\right)}{f^{n^{\prime}}(y)} d y^{\prime} \\
& =\frac{k\left(f^{n} y\right)}{\left|f^{n^{\prime}}(y)\right| k(y)} .
\end{aligned}
$$

By comparing (3.7), (3.8) and the Rohlin's formula of the hypothesis, we obtain

$$
\int \log \frac{q\left(y,\left[f^{-n} \eta\right](y)\right)}{p\left(y,\left[f^{-n} \eta\right](y)\right)} \bar{\mu}(d y)=0 .
$$

Consider the measure $\bar{\nu}$ defined on the $\sigma$-algebras of $\left[f^{-n} \eta\right]$ measurable sets by

$$
\bar{\nu}(B)=\int q(y, B) \bar{\mu}(d y) .
$$

(3.9) says that

$$
\int \log (d \bar{\nu} / d \bar{\mu}) d \bar{\mu}=0 .
$$

By the concavity of the function log, this is possible only if $\bar{\mu}=\bar{\nu}$, which implies that $q(y, B)=p(y, B)$ if $B$ is $f^{-n} \eta$ measurable.

As $f^{-n} \eta$ generates, the proposition follows by letting $n$ go to infinity.

Theorem 9 follows from proposition 3.6 because the partition $\eta$ is a subpartition of the partition $\xi$. Therefore, the Rohlin decomposition with respect to $\xi$ is made of measures which are linear combinations of measures from the Rohlin decomposition with respect to $\eta$. They are also absolutely continuous with respect to the Lebesgue measure.

\section{Proof of proposition 6}

We first recall some facts and remarks.

The estimation of the entropy from above, for any invariant measure, can be shown in various ways. The following result (4.1) is proved in [25].

Proposition 4.1. Let f be a $C^{1}$ map from I into itself and $\mu$ an invariant measure. Then $h(\mu) \leq \max \left(0, \int \log \left|f^{\prime}\right| d \mu\right)$.

In particular, proposition 4.1 explains why we called (1.1) a 'variational principle'.

To compute this entropy, we have to recall some formulae from [23]. Let $f$ be a C-map from $I$ into itself and $m$ a probability measure on $I$. If $\xi$ is a measurable partition, we denote by $E_{m}(/ \xi)(x)$ the Rohlin decomposition measure for $m$ with respect to $\xi$, at the point $x$. If $\varepsilon$ is the point partition, let us define

$$
H_{m}\left(\varepsilon / f^{-1} \varepsilon\right)=-\sum_{i} \int_{\left[a_{i}, a_{i+1}\right]} \log E_{m}\left(\left[a_{i}, a_{i+1}\right] / f^{-1} \varepsilon\right) d m .
$$

Proposition 4.2. For any invariant measure $\mu, h(\mu) \geq H_{\mu}\left(\varepsilon / f^{-1} \varepsilon\right)$.

We shall also use elementary properties of the function $m \rightarrow H_{m}\left(\varepsilon / f^{-1} \varepsilon\right)$. 
Proposition 4.3 (cf. [14] lemma 3.2). If $m=p m_{1}+(1-p) m_{2}$, then

$$
H_{m}\left(\varepsilon / f^{-1} \varepsilon\right) \geq p H_{m_{1}}\left(\varepsilon / f^{-1} \varepsilon\right)+(1-p) H_{m_{2}}\left(\varepsilon / f^{-1} \varepsilon\right) \text {. }
$$

If $m\left(\left\{a_{i}\right\}\right)=0$, and $m_{n}$ is a sequence of measures converging towards $m$, then

$$
H_{m}\left(\varepsilon / f^{-1} \varepsilon\right) \geq \lim \sup _{n} H_{m_{n}}\left(\varepsilon / f^{-1} \varepsilon\right) \text {. }
$$

Finally, let $f$ be a C-map, $\rho$ a positive function, and $B$ a Borel set. Suppose that $\int \rho d \lambda=1$. The Rohlin decomposition measures for $\rho \lambda$ with respect to $f^{-n} \varepsilon$ can be easily computed

$$
E_{\rho \lambda}\left(B / f^{-n} \varepsilon\right)(x)=\sum_{y \in f^{-n} f^{n} x} \frac{\rho(y) 1_{B}(y)}{\left|f^{n^{\prime}}(y)\right|} / \sum_{y \in f^{-n} f^{n} x} \frac{\rho(y)}{\left|f^{n^{\prime}}(y)\right|} \quad \text { (with } 0 / 0=0 \text { ). }
$$

We are now able to estimate $H_{\rho \lambda}\left(\varepsilon / f^{-n} \varepsilon\right)$. We have:

LEMMA 4.5. Let $f$ be a C-map, $\rho$ a positive function, $\int \rho d \lambda=1$. We have

$$
H_{\rho \lambda}\left(\varepsilon / f^{-n} \varepsilon\right) \geq \int \log \left|f^{n^{\prime}}\right| \rho d \lambda-\int \rho \log \rho d \lambda .
$$

Proof. Let us define $Q^{n}=\left\{Q_{i}^{n}\right\}$ the partition of $I$ defined by the critical points of $f^{n}$. By (4.4), we have:

$$
\begin{aligned}
H_{\rho \lambda}\left(\varepsilon / f^{-n} \varepsilon\right)= & \sum_{i} \int_{Q_{i}^{n}} \log \left[\left(\left|f^{n^{\prime}}(x)\right| \sum_{y \in f^{-n^{\prime} f^{n}}} \frac{\rho(y)}{\left|f^{n^{\prime}}(y)\right|}\right) / \rho(x)\right] \rho(x) d x \\
= & \int \log \left|f^{n^{\prime}}\right| \rho d \lambda-\int \rho \log \rho d \lambda \\
& +\int \log \sum_{y \in f^{-n} f^{n} x} \rho(y) /\left|f^{n^{\prime}}(y)\right| \rho(x) d x .
\end{aligned}
$$

By the change of variables formula, the last term is also equal to

$$
\int \log \sum_{y \in f^{n_{x}}} \frac{\rho(y)}{\left|f^{n^{\prime}}(y)\right|} \cdot \sum_{y \in f^{-n_{x}}} \frac{\rho(y)}{\left|f^{n^{\prime}}(y)\right|} d x .
$$

By convexity of the function $t \log t$, this quantity is greater than $\tau \log \tau$, where

$$
\tau=\int \sum_{y \in f^{-n_{x}}} \frac{\rho(y)}{\left|f^{n^{\prime}}(y)\right|} d x=\int \rho d \lambda=1 .
$$

Proof of proposition 6 . There is nothing to prove if there is almost surely no positive regular points. If that is not the case, there exists a measurable function $\rho, \int \rho d \lambda=1$, $\rho(x)>0$ only if $x$ is a positive regular point. We may also assume that $\rho$ is bounded and that $\rho \lambda$ integrates $\inf _{n}(1 / n) \log \left|f^{n^{\prime}}\right|$ so that $(1 / n) \log \left|f^{n^{\prime}}(x)\right|$ converges towards $\lambda_{x}$ in $L^{1}(I, \rho \lambda)$. Let $m_{n}$ be the measure $m_{n}=(1 / n) \sum_{i=0}^{n-1}(\rho \lambda) \circ f^{-i}$, and let us consider a measure $\mu$, vague limit point of the sequence $m_{n}$. We have, by $\mathrm{R}_{1}, \mu=\int \mu_{x} \rho(x) d x$, and that is the ergodic decomposition of the invariant measure $\mu$. We also have, by 
$\mathrm{R}_{2}$ and the choice of $\rho$, the convergence of $\int \log \left|f^{\prime}\right| d m_{n}=(1 / n) \int \log \left|f^{n^{\prime}}\right| d \rho \lambda$ towards $\int \lambda_{x} \rho(x) d x$. Therefore, we have:

$$
\begin{aligned}
H_{m_{n}}\left(\varepsilon / f^{-1} \varepsilon\right) & \geq(1 / n) \sum_{i=0}^{n-1} H_{\rho \lambda \circ f^{-i}}\left(\varepsilon / f^{-1} \varepsilon\right) \quad \text { by }(4.3), \\
& =(1 / n) \sum_{i=0}^{n-1} H_{\rho \lambda}\left(f^{-i} \varepsilon / f^{-i-1} \varepsilon\right)=(1 / n) H_{\rho \lambda}\left(\varepsilon / f^{-n} \varepsilon\right),
\end{aligned}
$$

by the sum formula for conditional entropies. Finally, by (4.5), we obtain

$$
H_{m_{n}}\left(\varepsilon / f^{-1} \varepsilon\right) \geq \int \log \left|f^{\prime}\right| d m_{n}-(\sup \rho \log \sup \rho) / n \text {. }
$$

If we had $\mu\left(\left\{a_{i}\right\}\right)>0$ for some critical point $a_{i}$, the corresponding ergodic component $\mu_{i}$ should verify $\int \log \left|f^{\prime}\right| d \mu_{i}=-\infty$, which contradicts $\mathbf{R}_{3}$. So, by (4.3) when $n$ goes to infinity, we have:

$$
H_{\mu}\left(\varepsilon / f^{-1} \varepsilon\right) \geq \lim \sup _{n} H_{m_{n}}\left(\varepsilon / f^{-1} \varepsilon\right) \geq \int \lambda_{x} \rho(x) d x
$$

And, by (4.2),

$$
\int h\left(\mu_{x}\right) \rho(x) d x=h\left(\mu_{x}\right) \geq H_{\mu}\left(\varepsilon / f^{-1} \varepsilon\right) \geq \int \lambda_{x} \rho(x) d x .
$$

Comparing this formula with (4.1) and $\mathrm{R}_{3}$ gives $h\left(\mu_{x}\right)=\lambda_{x} \rho \lambda$-almost everywhere, which is the statement of proposition 6 .

Let us remark that, in general, we can try to apply some limit argument and lemma 4.5 for constructing measures satisfying Rohlin's formula (1.1). Here $R_{3}$ was the condition which allowed us to control the behaviour of $(1 / n) \log \left|f^{n^{\prime}}\right|$. Replacing $R_{3}$ by another condition could be a way of finding invariant measures satisfying (1.1), and therefore by theorem 2 a.c.i.m.

\section{Non-degeneracy}

We prove in this section proposition 7. The arguments are not the same for a PC-map and an a.c.i.m. (lemma 5.1 and 5.2) as for a C-map and any invariant measure for which $\int \log \left|f^{\prime}\right|$ is finite (lemma 5.3 and 5.4).

LEMMA 5.1. Let $f$ be a PC-map and $\mu$ an a.c.i.m., with density $h$. Then $(1 / n) \log h\left(\Pi\left(f^{-n} y\right)\right)$ tends to $0 \bar{\mu}$-a.e.

Proof. Saying that $\mu=h \lambda$ is an invariant measure is equivalent to the relation

$$
h(x)=\sum_{x^{\prime} \in f^{-1} x} h\left(x^{\prime}\right) /\left|f^{\prime}\left(x^{\prime}\right)\right| .
$$

By lemma 4.4 we can compute

$$
I\left(\varepsilon / f^{-1} \varepsilon\right)(x)=-\sum_{i} 1_{Q_{i}}(x) \log E_{\mu}\left(1_{Q_{i}} / f^{-1} \varepsilon\right)(x)
$$


and find

$$
I\left(\varepsilon / f^{-1} \varepsilon\right)(x)=\log \left|f^{\prime}(x)\right|+\log h(f x)-\log h(x) .
$$

We have in the extension by applying $f^{-1}$

$$
\begin{aligned}
I(f \varepsilon / \varepsilon)(y) & =I\left(\varepsilon / f^{-1} \varepsilon\right)\left(f^{-1} y\right) \\
& =\log \left|f^{\prime}\left(f^{-1} y\right)\right|+\log h(\Pi(y))-\log h\left(\Pi\left(f^{-1} y\right)\right) .
\end{aligned}
$$

The lemma follows by lemma 3.5. We also have that the limit of the ergodic averages are the same for the functions $I(f \varepsilon / \varepsilon)$ and $\log \left|f^{\prime}\right|$.

Lemma 5.2. Let $f$ be a PC-map. Any a.c.i.m. is non-degenerate.

Proof. Let $a_{i}$ be a singular point and $\mu=h \lambda$ an a.c.i.m. The function

$$
k(x)=1 /\left(h(x) \sqrt{ }\left|x-a_{i}\right|\right)
$$

is $\mu$-integrable. Therefore we have

$$
\frac{1}{2} \log \left|x-a_{i}\right| \geq-\log h(x)-\log ^{+} k(x),
$$

with $\log ^{+} k \mu$-integrable. By lemma 5.1 and lemma 2.3 applied to $\log ^{+} k$, we have finally

$$
\lim _{n} \inf (1 / n) \log \left|\Pi\left(f^{-n} y\right)-a_{i}\right|=0 \quad \bar{\mu} \text {-a.e. }
$$

which proves lemma 5.2 .

LEMMA 5.3. Let $f$ be a C-map and $a_{i}$ a critical point for $f^{\prime}$. Let $\mu$ be an invariant measure on I such that $\int \log \left|f^{\prime}\right| d \mu$ is finite. Then

$$
\liminf _{n}(1 / n) \log \left|\Pi\left(f^{-n} y\right)-a_{i}\right|=0 \quad \bar{\mu} \text {-a.e. }
$$

Proof. Lemma 5.3 follows clearly from condition $\mathrm{C}_{3}$ and lemma 2.3 applied to the integrable function $\log \left|f^{\prime} \circ \Pi\right|$.

LEMMA 5.4. Let $f$ be $a$ C-map and $\mu$ an invariant measure without atoms and such that $\int \log \left|f^{\prime}\right| d \mu$ is finite. Then

$$
\lim _{n} \inf (1 / n) \log \left|\Pi\left(f^{-n} y\right)\right|=\lim _{n} \inf (1 / n) \log \left|1-\Pi\left(f^{-n} y\right)\right|=0 \quad \bar{\mu} \text {-a.e. }
$$

Proof. We may suppose $\mu$ ergodic and non-atomic. Let us remark first that the pre-image of 0 and 1 can be made only of critical points, and of 0 and 1 . We shall consider the different cases.

If $f^{-1}(\{0\})$ contains neither 0 nor 1 , every trajectory $\left\{f^{-n} y, n>0\right\}$ which would approach 0 exponentially fast on a subsequence would also by $C_{3}$ approach exponentially fast at the next stage another critical point. By (5.3) this happens only on a set of $\bar{\mu}$-measure 0 of initial points.

If $f^{-1}(\{0\})$ contains both 0 and 1 , the preceding remark applies to 1 . Therefore, if there is a positive set of points whose orbits approach 0 exponentially fast on a 
subsequence, almost all of them cannot approach exponentially fast another preimage of 0 other than 0 itself. We have here to study what happens in the neighbourhood of the fixed point 0 . There can exist non-constant orbits by $f^{-1}$ approaching 0 exponentially fast on a subsequence only if $f^{\prime}(0)>1$. Then, if the orbit cannot escape to another pre-image of 0 , it has to converge to 0 . Therefore the set of $y$ such that $\Pi\left(f^{-n} y\right)$ converges to 0 is of positive measure, and $\mu$ has to be the Dirac measure at 0 , which is impossible by hypothesis. So, in that case also, the set of points whose orbits approach 0 exponentially fast on a subsequence has measure zero. The same argument applies if $f^{-1}(\{0\})$ contains 0 and not 1 .

By exchanging the role of 0 and 1 , we are left only with the following case: $f^{-1}(\{0\})$ contains 1 and $f^{-1}(\{1\})$ contains 0 , where the same argument applies to $f^{2}$ instead of $f$; this achieves the proof of lemma 5.4.

\section{Conclusions}

We proved in the preceeding sections propositions 6 and 7 and theorems 8 and 9. The other results then follow.

Let us first show theorem 2. The formula for the entropy of an a.c.i.m. is due to Rohlin ([22], cf. also lemma 5.1 above for a proof). Conversely, let us consider for a C-map $f$ an ergodic invariant measure $\mu$ with positive entropy and satisfying formula (1.1). By proposition 7 this measure is non-degenerate, and we may apply theorem 9. Therefore for any subset $A$ of $I$ with Lebesgue measure zero, $\Pi^{-1}(A)$ is of $q(y, \cdot)$ measure zero for $\bar{\mu}$-almost every $y$. By averaging in $y, \Pi^{-1}(A)$ is of $\bar{\mu}$-measure zero, that is $\mu(A)=0$. The measure $\mu$ is absolutely continuous with respect to the Lebesgue measure.

We prove now theorem 5. Let $\mu$ be an a.c.i.m. Then $\log \left|f^{\prime}\right|$ is an integrable function (cf. again [22] or lemma 5.1). By the ergodic theorem applied to a countable dense subset of continuous functions and to $\log \left|f^{\prime}\right|, \mu$-almost every point is regular. Therefore, if $\mu=\int \mu_{x} \mu(d x)$ is the decomposition of the invariant measure $\mu$ into ergodic measures, we have:

$$
h(\mu)=\int h\left(\mu_{x}\right) \mu(d x)=\int \max \left(0, \lambda_{x}\right) \mu(d x) \quad \text { by proposition } 6 .
$$

If the entropy $h(\mu)$ is positive, the set of positive regular points has positive $\mu$-measure, and hence positive Lebesgue measure. Conversely, if the set of positive regular points has positive measure, there exists some $x_{0}$ to which proposition 6 applies and there exists some ergodic measure $\mu_{x_{0}}$ with

$$
h\left(\mu_{x_{0}}\right)=\lambda_{x_{0}}=\int \log \left|f^{\prime}\right| d \mu_{x_{0}}>0 .
$$

By theorem 2, $\mu_{x_{0}}$ is an a.c.i.m.

We then prove theorem 1. Let $f$ be a PC-map and $\mu$ an ergodic a.c.i.m. of positive entropy. By proposition 7 and theorem 9, the Rohlin decomposition measures for $\bar{\mu}$ with respect to the partition $\xi$, restricted to the $\sigma$-algebra $\Pi^{-1}(\mathscr{A})$, are all absolutely 
continuous with respect to the same measure. Let us remark that the partition $\xi$ is exactly the 'future' $\bigvee_{i=1}^{\infty} f^{i} Q$ of the partition $Q$ and that the $\sigma$-algebra $\Pi^{-1}(\mathscr{A})$ contains the $\sigma$-algebra generated by the 'past' $\sigma\left(f^{i} Q, i<0\right)$. That means that the $(Q, f, \bar{\mu})$ process has the following property: the Rohlin decomposition measures of $\bar{\mu}$ with respect to the future, restricted to the past, are all absolutely continuous with respect to the same measure. This property implies the statement of theorem 1 (Ratner [20], cf. also [13] and [17]). For completeness, we outline the argument here.

The Pinsker partition $\Pi_{Q}$ of the process is contained in both the future and the past. The property above implies that the Rohlin decomposition measures of $\bar{\mu}$ with respect to $\Pi_{Q}$, restricted to the Pinsker $\sigma$-algebra $\sigma\left(\Pi_{Q}\right)$ itself, are all absolutely continuous with respect to the same measure. This is possible only if $\Pi_{Q}$ is countable.

By ergodicity, a countable invariant partition is finite and its elements are ergodic sets for a suitable power $f^{k_{0}}$. As the Pinsker partition for the $\left(\bigvee_{i=0}^{k_{0}-1} f^{k_{0}} Q, f^{k_{0}}\right)$ process is the same as the one for the $(Q, f)$ process, we obtain all the conclusions of the theorem if we show that $\Pi_{Q}$ trivial and the property of the decomposition imply weak Bernoulli. But if the Pinsker partition $\Pi_{Q}$ is trivial, we have that the terms in the Rohlin decomposition for the measure $\bar{\mu}$ with respect to the future of the partition $Q$, restricted on the remote past $\sigma$-algebra $\bigwedge_{n} \sigma\left(f^{-i} Q, i \geq n\right)$, are all absolutely continuous with respect to the same measure - and also have an average by $\bar{\mu}$ which takes only the values 0 and $1\left(\right.$ for $\left.\bigwedge_{n} \sigma\left(f^{-i} Q, i \geq n\right)=\Pi_{Q} \bar{\mu} \bmod 0\right)$. Therefore these restrictions to the remote past $\sigma_{-}$-algebra must all coincide. This condition - that Rohlin decomposition measures with respect to the future coincide on the remote past - is another form of the weak Bernoulli condition we gave in $\S 1$, as can be seen by applying $f^{-m}$, letting $m$ and $p$, and then $n$ go to infinity.

Corollaries 2 and 4 are deduced in the same way. Let us consider a PC-map $f$ and an ergodic a.c.i.m. $\mu$. If $\int \log \left|f^{\prime}\right| d \mu=0$, we have, by Rohlin's formula, $h(\mu)=0$ and the map $f$ is $\mu$-a.e. invertible; in other words, $f^{-1} \mathscr{A}=\mathscr{A} \bmod 0$. We also have $f^{-n} \mathscr{A}=\mathscr{A}$ for all $n$. Conversely, if $\int \log \left|f^{\prime}\right| d \mu>0$, as we have $h(\mu)=H_{\mu}\left(\varepsilon / f^{-1} \varepsilon\right)$, the $\sigma$-algebra $\bigwedge_{n} f^{-n} \mathscr{A}$ is the Pinsker $\sigma$-algebra $\sigma(\Pi)$. But for the same reason, by 3.2 (ii), $\sigma(\Pi)$ is also included in the $\sigma$-algebra $\sigma(\eta)$. From proposition 3.5 and the argument above, it follows that the $\sigma$-algebra $\sigma(\Pi)$ is fin:te, and that proves corollary 2 .

Let us now consider a PC-map $f, \mu$ an a.c.i.m. and $\mu=\int \mu_{x} \mu(d x)$ its ergodic decomposition. By proposition $7 \mu$ is non-degenerate and therefore almost all $\mu_{x}$ are non-degenerate. 
In addition, the proof of lemma 5.1 gives us, if we identify the limits, the following relation: $h\left(\mu_{x}\right)=\int \log \left|f^{\prime}\right| d \mu_{x}$ for almost all $\mu_{x}$. Theorem 2 applied to a PC-map and a non-degenerate measure gives that almost all $\mu_{x}$ with positive entropy has to be an a.c.i.m., which is the statement of corollary 4 .

\section{REFERENCES}

[1] G. Benettin, M. Casartelli, L. Galgani, A. Giorgilli \& J.-M. Strelcyn. On the reliability of numerical studies of stochasticity. II. Identification of time averages. Il nuovo Cimento 50 B (2) (1979), 211-232.

[2] R. Bowen. A horseshoe with positive measure. Inventiones Math. 29 (1975), 203-204.

[3] R. Bowen. Bernoulli maps of the interval. Israel J. Maths 28 (1977), 161-168.

[4] R. Bowen. Invariant measures for Markov maps of the interval. Commun. Math. Phys. 69 (1979), 1-18.

[5] R. Bowen \& D. Ruelle. The ergodic theory of axiom $A$ flows. Inventiones Math. 29 (1975), 181-202.

[6] L. A. Bunimovic \& Y. G. Sinaī. Stochastic attractors and Lorenz model. In Nonlinear Waves (in Russian) Nauka Press, 1979.

[7] A. Fathi, M. Herman \& J.-C. Yoccoz. Seminar notes, in preparation.

[8] F. Hofbauer. Maximal measures for simple piecewise monotonic transformations. $Z$. Wahrscheinlichkeitstheorie verw. Gebiete 52 (1980), 289-300.

[9] K. Jacobs. Lecture Notes on Ergodic Theory. Aarhus Universitët, 1963.

[10] M. V. Jakobson, Construction of invariant measures absolutely continuous with respect to $d x$ for some maps of the interval. In Global Theory of Dynamical Systems. Springer Lecture Notes in Math. no. 819. Springer: Berlin, 1980.

[11] A. Katok \& J.-M. Strelcyn. Invariant manifold for smooth maps with singularities. Preprint.

[12] F. Ledrappier. Principe variationnel et systèmes dynamiques symboliques. $Z$. Wahrscheinlichkeitstheorie verw. Gebiete 30 (1974), 185-202.

[13] F. Ledrappier. Sur la condition de Bernoulli faible et ses applications. Théorie ergodique; Rennes 73/74. Springer Lecture Notes in Math. no. 532. Springer: Berlin, 1976.

[14] F. Ledrappier \& P. Walters. A relativised variational principle for continuous transformations. $J$. London Math. Soc. (2) 16 (1977), 568-576.

[15] M. Misiurewicz. Absolutely continuous measures for certain maps of an interval. Preprint: IHES, 1979.

[16] D. S. Ornstein. Ergodic Theory, Randomness and Dynamical Systems. Yale University Press: New Haven, 1974.

[17] D. S. Ornstein \& B. Weiss. Geodesic flows are Bernoüllian. Israel J. Math. 14 (1973), 184-198.

[18] Y. B. Pesin. Families of invariant manifolds corresponding to non-vanishing characteristic exponents. Izv. Akad. Nauk. SSSR Ser. Mat. 40 (1976), 1332-1379. Math USSR Izv. 10 (1976), 1261-1305.

[19] Y. B. Pesin. Description of the II-partition of a diffeomorphism with invariant smooth measure. Mat. Zametki 21(6) (1977), 29-44.

[20] M. Ratner. Anosov flows are also Bernoullian. Israel J. Math. 17 (1974), 380-391.

[21] V. A. Rohlin. On the fundamental ideas in measure theory. Mat. Sb. 25 (1949), 107-150. Amer. Math. Soc. Trans. (1) 10 (1962), 1-54.

[22] V. A. Rohlin. Exact endomorphism of a Lebesgue space. Izv. Akad. Nauk. SSSR Ser. Mat. 25 (1961), 499-530. Amer. Math. Soc. Trans. (2) 39 (1964), 1-36.

[23] V. A. Rohlin. Lectures on the entropy theory of transformations with an invariant measure. Usp. Mat. Nauk. 22 (5) (1967), 3-56. Russian Math. Surveys 22(5) (1967), 1-52.

[24] D. Ruelle. A measure associated with axiom $A$ attractors. Amer. J. Math. 98 (1975), 289-294.

[25] D. Ruelle. An inequality for the entropy of differentiable maps. Bol. Soc. Bras. Mat. 9 (1978), 83-87.

[26] D. Ruelle. Sensitive dependence on initial conditions and turbulent behaviour of dynamical systems. Ann. N.Y. Acad. Sci. 316 (1978), 408-416.

[27] D. Ruelle. Ergodic theory of differentiable dynamical systems Publ. math. IHES 50 (1979), $27-58$. 
[28] D. Ruelle. Characteristic exponents and invariant manifolds in Hilbert space. Preprint: IHES, 1980.

[29] D. Ruelle \& M. Shub. Stable manifolds for maps. In Global Theory of Dynamical Systems. Springer Lecture Notes in Math. no. 819. Springer: Berlin, 1980.

[30] Y. G. Sinaī. Classical systems with countable Lebesgue spectrum II. Izv. Akad. Nauk. SSSR Ser. Mat. 30 (1966), 15-68. Amer. Math. Soc. Trans (2) 68 (1968), 34-88.

[31] Y. G. Sinaī. Markov partitions and C-diffeomorphisms. Funk. Analiz i Pri. 2 (1968), 61-82. Func. Ana. Appl. 2 (1968), 64-89.

[32] Y. G. Sinaĩ. Gibbs measure in ergodic theory. Uspehi Mat. Nauk 27(4) (1972), 21-63. Russian Math. Surveys 27(4) (1972), 21-69.

[33] W. Szlenk. Some dynamical properties of certain mappings of an interval, part I and II. Preprint: IHES, 1980.

[34] P. Walters. Equilibrium states for $\beta$-transformations and related transformations. Math. Z. 159 (1978), 65-88. 